processing before being secreted as the mature form, but several pathogenic SFTPC mutations associated with familial interstitial lung disease impede this process. Mutations in the C-terminal BRICHOS domain of SFTPC ( $\triangle \mathrm{Ex} 4$ and L188Q) lead to retention of the protein within the endoplasmic reticulum (ER), while other mutations (e.g. I73T) cause SFTPC mis-trafficking.

Methods To study these mutants in vivo in a genetically tractable organism, we generated lines of Drosophila melanogaster expressing wild type or mutant human SFTPC. The transgenic proteins could be tagged with green fluorescent protein (GFP) to facilitate in vivo visualisation. These fusion proteins were expressed under the control of tissue-specific drivers. Components of the ER associated degradation (ERAD) machinery or of the autophagy pathway were depleted in those tissues by RNA interference. Lines expressing an ER stress reporter or autophagy reporter were used as readouts for these phenomena.

Results Expression of the BRICHOS mutants $\triangle \mathrm{Ex} 4$ and L188Q led to the progressive deposition of protein aggregates when expressed in the fly eye. In contrast, the I73T mutant accumulated in a more diffuse distribution. When expressed in the larval salivary gland, the BRICHOS mutants where retained within the cell, in contrast to the wild type protein that was trafficked to the cell surface. The I73T mutant showed low-level cell surface and weak intracellular fluorescence. Depletion of the ERAD E3 ubiquitin ligase Hrd1 or its associated E2 ligases failed to affect mutant protein levels arguing against an important role of ERAD in the degradation of SFTPC in this model. In contrast, inhibition of autophagy by depletion of Atg8 enhanced the accumulation of L188Q SFTPC. Accordingly, robust activation of autophagy was detected in L188Q SFTPC-expressing tissue. Interestingly, ER stress was not detected.

Conclusion In a Drosophila model of hSFTPC trafficking, autophagy was the major degradation pathway for L188Q mutant SFTPC.

\section{S63 HUMAN MESENCHYMAL STROMAL CELL (HMSC) REGULATION OF HUMAN MACROPHAGES IN IN VITRO MODELS OF THE ACUTE RESPIRATORY DISTRESS SYNDROME (ARDS)}

T Morrison, M Jackson, A Kissenpfennig, C O'Kane, D McAuley, A Krasnodembskaya. Queen's University Belfast, Belfast, UK

\subsection{6/thoraxjnl-2015-207770.69}

Background Currently there is no effective therapy which targets the mechanisms underlying the development of ARDS. MSCs present a promising candidate therapy and are being tested in clinical trials for ARDS however their mechanisms of effect in ARDS are not fully understood. Since the alveolar macrophage is key to orchestrating the alveolar inflammatory response, it was hypothesised that hMSCs induce an anti-inflammatory M2-like phenotype in human macrophages. The aim of this study therefore was to determine the effect of MSCs on macrophage phenotype and function and to elucidate the mechanisms of these effects.

Methods Using an in vitro non-contact co-culture system, human MSCs and human monocyte-derived-macrophages (MDMs) were stimulated with E.coli lipopolysaccharide (LPS). Cytokine and marker expression profiles were examined using ELISAs, multiplex and flow cytometry. Phagocytic capacity of MDMs was measured using fluorescent E.coli bioparticles by flow cytometry. For additional clinical relevance, the ARDS microenvironment was mimicked by using bronchoalveolar lavage fluid (BALF) obtained from patients with ARDS to examine the effect of MSCs.
Results MSCs suppress the production of both pro-inflammatory and anti-inflammatory cytokines by MDMs stimulated with LPS. MSCs increase expression of M2 markers CD163 and CD206 and have no effect on M1 markers CD80 and ICAM-1. Importantly, in spite of the immunosuppressive effect on macrophages, MSCs increase their phagocytic capacity. MSC effects on cytokine secretion and marker expression were maintained in the presence of BALF from patients with ARDS (Figure 1).

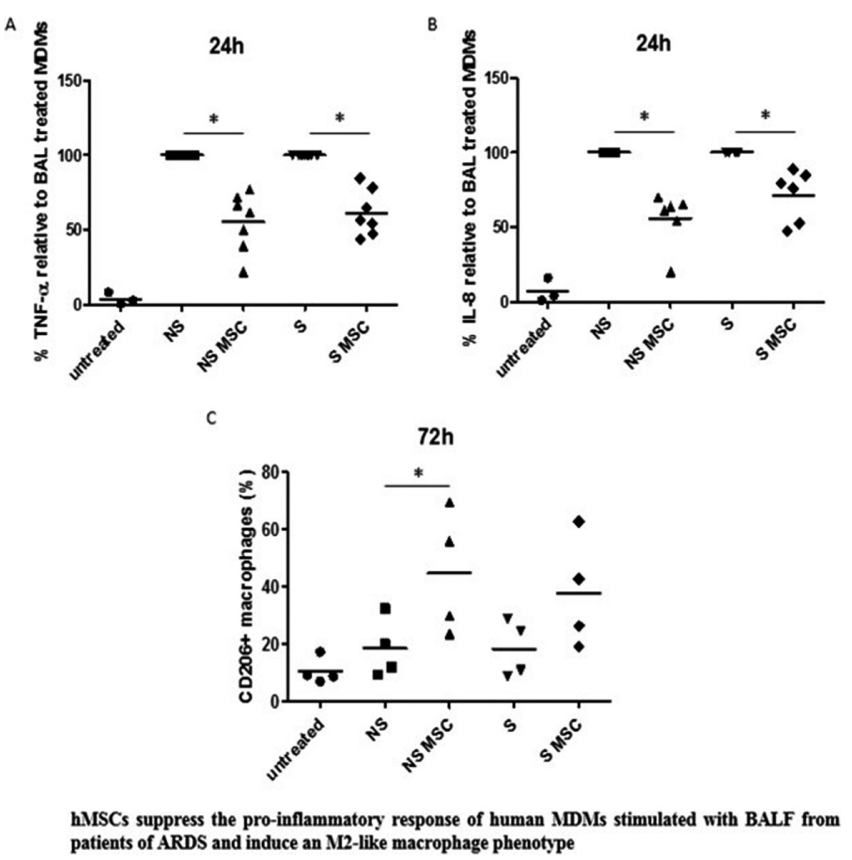

Abstract S63 Figure 1 MSCs decrease secretion of pro-inflammatory cytokines TNF- $\alpha$ (A) and IL-8 (B) and increase expression of M2 macrophage marker CD206 (C) by MDMs stimulated with BALF from non-septic (NS) or septic (S) patients of ARDS. (A + B, n = 3-7, Kruskal Wallis * $p<0.05)(C, n=4$, ANOVA * $p<0.05)$

Conclusions Human bone marrow-derived MSCs induce an M2like phenotype and suppress cytokine secretion in primary human MDMs stimulated with LPS or ARDS patient BALF. Importantly, these effects are coupled with augmentation of macrophage phagocytosis which may be important in the clearance of bacteria and apoptotic cells. Uncovering the paracrine mechanisms responsible for the MSC effects on human macrophages remain the focus of ongoing work.

Supported by MRC MR/L017229/1, Department of Employment and Learning.

Some of the materials employed in this work were provided by the Texas A $\circlearrowleft$ M Health Science Centre College of Medicine Institute for Regenerative Medicine at Scott and White through a grant from NCRR of the NIH, Grant \# P40RR017447.

\section{S64 ALVEOLAR EPITHELIAL TYPE II CELL EXPRESSION OF VEGF-AXXXA IS CRITICAL FOR DEVELOPMENT OF IDIOPATHIC PULMONARY FIBROSIS (IPF): AN ANTI- FIBROTIC ROLE FOR VEGF-AXXXB ANTI-ANGIOGENIC ISOFORMS?}

${ }^{1} \mathrm{SL}$ Barratt, ${ }^{1} \mathrm{~T}$ Blythe, ${ }^{1} \mathrm{C}$ Jarrett, ${ }^{1} \mathrm{~K}$ Ourradi, ${ }^{1} \mathrm{G} I$ Welsh, ${ }^{2} \mathrm{C}$ Scotton, ${ }^{3} \mathrm{DO}$ Bates, ${ }^{1} \mathrm{AB}$ Millar. ${ }^{1}$ University of Bristol, Bristol, UK; ${ }^{2}$ University of Exeter, Exeter, UK; ${ }^{3}$ University of Nottingham, Nottingham, UK

10.1136/thoraxjnl-2015-207770.70 